\title{
POSITIVE ENERGY REPRESENTATIONS FOR QUANTUM SPIN MODELS IN $1+1$ DIMENSIONS
}

\author{
TAKU MATSUI \\ Graduate School of Mathematics \\ Kyushu University, Hakozaki, Fukuoka, Japan \\ E-mail:matsui@math.kyushu-u.ac.jp
}

\begin{abstract}
We present recent results on positive energy representations of quantum spin models.

1. Introduction. In this article, we review recent results on positive energy representations for quantum models on the $1+1$ dimensional space time. Our aim is to give a complete list of positive energy representations.

Let $\mathcal{A}$ be a unital $C^{*}$-algebra and $\alpha_{t}$ be a one-parameter group of automorphisms of $\mathcal{A}$. We call the pair $\left\{\mathcal{A}, \alpha_{t}\right\}$ a $C^{*}$-dynamical system and $\alpha_{t}$ is referred to as the time evolution.
\end{abstract}

Definition 1.1. Let $\left\{\mathcal{A}, \alpha_{t}\right\}$ be a $C^{*}$-dynamical system. A representation $\pi$ of $\mathcal{A}$ on a Hilbert space $\mathcal{H}$ is a positive energy representation for $\left\{\mathcal{A}, \alpha_{t}\right\}$ iff there exists a positive selfadjoint operator $H_{\pi}$ such that the unitary group generated by $H_{\pi}$ implements the time evolution $\alpha_{t}$.

$$
e^{i t H_{\pi}} \pi(Q) e^{-i t H_{\pi}}=\pi\left(\alpha_{t}(Q)\right)
$$

Physically speaking, the spectrum of the generator of the time evolution is identified with the energy of a physical state. The positivity of the spectrum is related to the stability of the matter. In the algebraic approach to quantum field theory $([11])$, the positivity of the spectrum of the Hamiltonian is one of basic requirements for selection of representations of the algebra of the physical local observables. The equivalence classes of positive energy representations are called sectors. Apart from standard sectors such as vacuum representations (with space time covariance and vacuum vector) and charged sectors (dual objects of gauge symmetry), we have two types of exotic sectors, soliton

1991 Mathematics Subject Classification: Primary 46C20; Secondary 32G81.

Research supported by Sumitomo Foundation, Japan.

The paper is in final form and no version of it will be published elsewhere. 
sectors and infrared representations. Infrared representations arises in massless field theories. They consist of infinitely many small energy states which produce representations disjoint from vacuum sectors. Soliton sectors exist when more than two vacuum sectors are present. A soliton sector is the interface of these two different vacuum sectors. The construction of soliton sectors was done in $P(\phi)_{2}$ models by J. Frölich (cf. [8]). In that example, the internal symmetry is broken and the different vacuum sectors are connected by the gauge transformation. Recently D. Schlingemann obtained the general construction of soliton sectors for two dimensional models where different vacuum sectors are not necessarily connected by the gauge symmetry (cf. [17]).

On the other hand, for electrons with a long range interaction (e.g. coulomb forces), the positivity requirement of the spectra may be too restrictive. It might be sufficient that the system is stable thermodynamically.

Our motivation for studying positive energy representations originated from recent results of the kink (static soliton) for the XXZ model (see $[2,9,10,12,3,14,15,16]$ ). The existence and an explicit description of the static kink were established in $[2,9,10]$, and a complete list of positive energy representations is obtained in certain cases $([3,14])$. We consider quantum lattice models on the integer lattice $\mathbf{Z}$. We use the mathematical framework for quantum lattice models presented in [6]. The algebra of quantum observables is the UHF $C^{*}$-algebra $\mathcal{A}$ (the infinite tensor product of the algebra $M_{n}(\mathbf{C})$ of $n \times n$ complex matrices)

$$
\mathcal{A}=\bigotimes_{\mathbf{z}} M_{n}(\mathbf{C})
$$

where the component of the tensor product is indexed by an integer $j$. Let $Q$ be a matrix in $M_{n}(\mathbf{C})$. By $Q^{(j)}$ we denote the one site observable $Q$ located at $j$. Given a subset $\Lambda$ of $\mathbf{Z}, \mathcal{A}_{\Lambda}$ is defined as the subalgebra of $\mathcal{A}$ generated by all $Q^{(j)}$ with $Q \in M_{n}(\mathbf{C}), j \in \Lambda$. We also set

$$
\mathcal{A}_{\text {loc }}=\bigcup_{|\Lambda|<\infty} \mathcal{A}_{\Lambda}
$$

where $|\Lambda|$ is the cardinality of $\Lambda$. Suppose that $\varphi$ is a state on $\mathcal{A}$. The restriction of $\varphi$ to $\mathcal{A}_{\Lambda}$ is denoted by $\varphi_{\Lambda}$

$$
\left.\varphi\right|_{\mathcal{A}_{\Lambda}}=\varphi_{\Lambda}
$$

The lattice translation $\tau_{j}$ is an automorphism of $\mathcal{A}$ defined by $\tau_{j}\left(Q^{(k)}\right)=Q^{(j+k)}$.

The time evolution $\alpha_{t}$ of our systems is generated by the translation invariant finite range Hamiltonian. This means that we have a selfadjoint local energy operator $h_{0}$ such that $h_{0}=h_{0}^{*} \in \mathcal{A}_{\Lambda_{0}}$ where $\Lambda_{0}$ is a finite subset of $\mathbf{Z}$ which contains the origin 0 . The radius of $\Lambda_{0}$ is called the range of the interaction. We set $\tau_{j}\left(h_{0}\right)=h_{j}$. The finite volume Hamiltonian $H_{\Lambda}$ is determined by

$$
H_{\Lambda}=\sum_{j: j+\Lambda_{0} \subset \Lambda} h_{j}
$$

while the formal infinite volume Hamiltonian will be denoted by

$$
H=\sum_{j \in \mathbf{Z}} h_{j} .
$$


The time evolution $\alpha_{t}(Q)$ of $Q \in \mathcal{A}$ is obtained via the thermodynamic limit

$$
\alpha_{t}(Q)=\lim _{\Lambda \rightarrow \mathbf{Z}} e^{i t H_{\Lambda}} Q e^{-i t H_{\Lambda}} .
$$

The generator $\delta$ of $\alpha_{t}$ is denoted by $\delta(Q)=i[H, Q]$. It is known that $\mathcal{A}_{\text {loc }}$ is a core of $\delta$. The typical example for analysis is the spin $1 / 2 \mathrm{XXZ}$ model where the Hamiltonian is determined by

$$
H=-\sum_{j \in \mathbf{Z}}\left\{\Delta \sigma_{z}^{(j)} \sigma_{z}^{(j+1)}-\left(\sigma_{x}^{(j)} \sigma_{x}^{(j+1)}+\sigma_{y}^{(j)} \sigma_{y}^{(j+1)}\right)\right\}
$$

where $\Delta$ is a real parameter and $\sigma_{x}, \sigma_{y}$ and $\sigma_{z}$ are Pauli spin matrices.

We will see that the outlook of the set of all positive energy representations is quite similar to that of quantum field theory.

2. Ground states. We begin with the notion of the ground state representation. Consider a positive energy representation $\{\pi, \mathcal{H}\}$. If the bottom of the spectrum of $H_{\pi}$ is a point spectrum we call $\{\pi, \mathcal{H}\}$ the ground state representation, and the unit eigenvector for the least eigenvalue is called the ground state vector. In terms of the state associated with a ground state vector, we arrive at the following definition

Definition 2.1. A state $\varphi$ is a ground state if and only if

$$
\varphi\left(Q^{*}[H, Q]\right)=\lim _{\Lambda \rightarrow \mathbf{Z}} \varphi\left(Q^{*}\left[H_{\Lambda}, Q\right]\right) \geq 0
$$

for any $Q \in \mathcal{A}_{\text {loc }}$.

The GNS representation of a ground state satisfying (2.1) gives rise to a ground state representation.

The standard construction of the ground state for quantum spin models is to take the thermodynamic limit of the finite volume ground states in the following sense. As $H_{\Lambda}$ is a strictly local element, we can regard it as a finite matrix. Take a unit eigenvector $\Omega_{\Lambda}$ with the smallest eigenvalue and consider the vector state $\omega_{\Lambda}$ of $\mathcal{A}_{\Lambda}$ associated with $\Omega_{\Lambda}$

$$
\omega_{\Lambda}(Q)=\left(\Omega_{\Lambda}, Q \Omega_{\Lambda}\right), \quad Q \in \mathcal{A}_{\text {loc }} .
$$

Take $\Lambda=[n, m]$ and consider the limit

$$
\lim _{\substack{n \rightarrow-\infty \\ m \rightarrow+\infty}} \omega_{\Lambda}(Q)=\omega_{\infty}(Q) .
$$

The state thus obtained is a ground state in the sense specified above. This procedure contains some ambiguity. The first point is that the ground state vector for finite volume may not be unique. The second point is that the thermodynamic limit may not exist. Instead of the limit (2.2), we may take the limit of subsequences and still we obtain a ground state. Mathematical physicists often claim that the uniqueness of the ground state follows from the uniqueness of the finite volume ground state and the existence of the thermodynamic limit but this is not the uniqueness of the ground state in the sense of Definition 2.1. An example which clarifies the difference of these two claims is the quantum Ising model. The model is exactly solvable and the Hamiltonian is given by

$$
H=-\sum_{j \in \mathbf{Z}} \sigma_{z}^{(j)} \sigma_{z}^{(j+1)}-2 \lambda \sum_{k \in \mathbf{Z}} \sigma_{x}^{(k)} .
$$


If we choose the basis for which $\sigma_{z}$ is diagonal and $\sigma_{x}$ is off diagonal and real, then $-H_{\Lambda}$ satisfies the condition of Perron Frobenius Theorem and the finite volume ground state is unique. It is also easy to verify that the thermodynamic limit (2.2) exists, however, the infinite volume ground state is not unique when $|\lambda|<1$. The ground state, in the sense of Definition 2.1, is unique for $|\lambda| \geq 1$ (see [4]). In fact the gap between the first and the second eigenvalue of $H_{\Lambda}$ converges to zero exponentially fast in the thermodynamic limit if $|\lambda|<1$ and the gap is open uniformly in $\Lambda$ if $|\lambda|>1$.

A slightly different way of construction of (infinite volume) ground states is adding boundary terms. In the case of the XXZ model (1.4), some physicists consider the following "boundary magnetic fields"

$$
\tilde{H}_{\Lambda}=H_{\Lambda}+\sum_{j \in \partial \Lambda} \lambda_{j} \sigma_{z}^{(j)}
$$

where $\lambda_{j}$ is a real number dependent on the site $j$. The above model with an additional boundary term is solvable but the structure of eigenstates may change according to the value $\Delta$. The thermodynamic limit of the ground state for $\tilde{H}_{\Lambda}$ is a ground state (again in the sense of Definition 2.1). For example, consider the case when the model is ferromagnetic, $\Delta>1$ and $\Lambda=[n, m]$. The ground state of $\tilde{H}_{\Lambda}$ is one-dimensional if $\lambda_{n} \lambda_{m}>0$. It is two-dimensional if $0 \leq \lambda_{n}=-\lambda_{m}<\sqrt{\Delta^{2}-1}$. The dimension of the ground state is proportional to $|\Lambda|$ when $\lambda_{n}=-\lambda_{m}=\sqrt{\Delta^{2}-1}$ where this finite system obtains the quantum group $S U_{q}(2)$ symmetry. In this way we obtain both translation invariant ground states and non periodic ground states. (We will return to this model later.)

Thus we generalize the above construction of the ground state. Suppose that a selfadjoint element $B_{\Lambda}$ in $\mathcal{A}_{\Lambda}$ satisfies the following condition

$$
\lim _{\Lambda \rightarrow \mathbf{Z}}\left[H_{\Lambda}+B_{\Lambda}, Q\right]=\lim _{\Lambda \rightarrow \mathbf{Z}}\left[H_{\Lambda}, Q\right]
$$

for all $Q \in \mathcal{A}_{\text {loc }}$. Consider the finite volume ground states for $H_{\Lambda}+B_{\Lambda}$, say, $\omega_{\Lambda}$ and again take the limit (2.2). We obtain again a state satisfying the condition of Definition 2.1.

Related to ground states, a notion frequently used in mathematical physics is "zero energy state". A state $\varphi$ of $\mathcal{A}$ is a zero energy state if and only if for any $j$ in $\mathbf{Z}$

$$
\varphi\left(h_{j}\right)=\inf \left\{\psi\left(h_{0}\right)\right\}
$$

where the infimum is taken among all states $\psi$. The zero energy state may not exist in general. The zero energy state is automatically a ground state satisfying (2.1). Some examples of Hamiltonian with zero energy states are the XXZ model (1.4) with $\Delta \geq 1$ and the AKLT model (cf. [1]) and its extension (cf. [7]). Physicists may claim that the model with zero energy state is artificial as it neglects quantum frustration and fluctuation appearing in antiferromagnetic systems.

Positive energy representations can be constructed as a bounded perturbation of the ground state representation. Suppose a ground state $\omega$ of the perturbed Hamiltonian $H^{\prime}$ is given where $H^{\prime}=H+B . H$ is the considered Hamiltonian and $B$ is any selfadjoint element of $\mathcal{A}$. The GNS representation associated with $\omega$ has a positive energy. Note that the point spectrum may be unstable under bounded perturbation even if the norm of the perturbing operator is small so that a positive energy representation constructed by 
perturbation of a ground state representation may fail to be another ground state.

It is interesting to know whether all the ground states or positive energy representations of quantum spin systems are constructed in this way. We do not have the complete answer to this question, however we have results under some additional assumption which we will explain later.

We next state standard results on ground states and positive energy representations. The first is the variation principle. The following theorem is due to O. Bratteli, A. Kishimoto and D. Robinson (see [5]).

Theorem 2.2 (Bratteli, Kishimoto, Robinson). Let $\varphi$ be a state on $\mathcal{A}$. The following conditions are equivalent:

(i) $\varphi$ is a ground state;

(ii) For any integers $n$ and $m(n<m)$

$$
\varphi\left(H_{[n, m]}+B_{[n, m]}\right)=\inf \psi\left(H_{[n, m]}+B_{[n, m]}\right)
$$

where the infimum is taken over all states $\psi$ satisfying

$$
\varphi_{[n, m]^{c}}=\psi_{[n, m]^{c}} .
$$

To obtain the above theorem, O. Bratteli, A. Kishimoto and D. Robinson used the following lemma and the theorem of Borchers.

Lemma 2.3. Suppose that $\Lambda$ is a finite subset of $\mathbf{Z}$ and $\varphi$ and $\psi$ are states of $\mathcal{A}$. If these are identical outside $\Lambda$, i.e. $\varphi_{\Lambda^{c}}=\psi_{\Lambda^{c}}$, then the GNS representations associated with $\varphi$ and $\psi$ are quasi-equivalent. In particular, when both $\varphi$ and $\psi$ are pure, they are unitarily equivalent.

The theorem of Borchers tells us that we can redefine $H_{\pi}$ which is affiliated to the von Neumann algebra $\pi(\mathcal{A})^{\prime \prime}$ generated by $\pi(\mathcal{A})$ (cf. Theorem 3.2.46 in [6]).

An application of the idea of the proof of Theorem 2.2 leads to the following inequality for our $1+1$ dimensional models

LEMMA 2.4. Let $\pi$ be a positive energy representation for the Hamiltonian (1.3) on the Hilbert space $\mathcal{H}$. Let $\eta$ be in the domain of $H_{\pi}$. Then for $\Lambda=[n, m]$

$$
\left(\eta, \pi\left(H_{\Lambda}\right) \eta\right) \leq \inf \operatorname{spec}\left(H_{\Lambda}\right)+\left(\eta, H_{\pi} \eta\right)+2 r\left\|h_{0}\right\|
$$

where $\inf \operatorname{spec}\left(H_{\Lambda}\right)$ is the infimum of the spectrum of $H_{\Lambda}$ and $r$ is the range of interaction.

For simple cases such as the ferromagnetic XXZ model, the set of all ground states and positive energy representations is determined by an application of the above theorem and lemmas. Simplicity originates from the fact that the pure ground states are product states or equivalent to product states. (We say two states are equivalent if their GNS representations are unitarily equivalent.) We do not have satisfactory results to obtain a complete list of positive energy representations. On the other hand, in the next section we will see that the Hamiltonian with gapless spectrum possesses uncountably many positive energy (infrared) representations which are not ground state representations. Probably these representations are not physically meaningful.

For the Hamiltonian with spectral gap, we will present a partial answer to classification of positive energy and ground state representations in Section 4. 
3. Infrared representations. We now consider infrared representations. As is stated briefly in Section 1, the infrared representation appears as an exotic positive energy representation in the massless quantum field theory. A state in infrared representation can be constructed as follows.

Consider a ground state representation $\pi$ on $\mathcal{H}$ and the positive Hamiltonian $H_{\pi}$ on $\mathcal{H}$. Let $\Omega$ be a ground state vector for $H_{\pi}$. Suppose that the spectrum of $H_{\pi}$ is gapless, i.e. $\operatorname{spec}\left(H_{\pi}\right) \cap(0, \varepsilon) \neq \emptyset$ for any small $\varepsilon>0$. Take a sequence of positive numbers $\left\{\varepsilon_{1}, \varepsilon_{2}, \varepsilon_{3}, \ldots\right\}$ such that the sum is finite: $E=\sum_{j} \varepsilon_{j}<\infty$. Take mutually orthogonal unit vectors $\eta_{j}$ with energy smaller than $\varepsilon_{j}$. Let $A_{j}$ be a creation operator for a (quasi-)particle carrying energy smaller than $\varepsilon_{j}$ such that $A_{j} \Omega=\eta_{j}$. Next, consider the state $\varphi$ obtained by the following weak* limit.

$$
\varphi(Q)=\lim _{j \rightarrow \infty} \frac{\left(\prod_{k=1}^{j} A_{k} \Omega, Q \prod_{k=1}^{j} A_{k} \Omega\right)}{\left\|\prod_{1}^{j} A_{k} \Omega\right\|^{2}} .
$$

The state $\varphi$ has a finite energy smaller than $E$. But as infinitely many particles are created to produce $\varphi$, it is natural to expect that $\varphi$ is not equivalent to the ground state originally given. On the other hand, if there is no bound state $\left(H_{\pi}\right.$ has only continuous spectra), we may expect that $\varphi$ is not invariant under the time evolution $\alpha_{t}$. Thus we may conclude $\varphi$ is not a ground state but a positive energy state.

In general this heuristic argument may not work, however, for free massless bosons and fermions we can construct the infrared representation precisely in this way. The same construction works for the XY model (see [3]). The Hamiltonian of the XY model is determined by

$$
H=\sum_{j \in \mathbf{Z}}\left\{(1+\gamma) \sigma_{x}^{(j)} \sigma_{x}^{(j+1)}+(1-\gamma) \sigma_{y}^{(j)} \sigma_{y}^{(j+1)}\right\}+2 \lambda \sum_{j} \sigma_{z}^{(j)}
$$

where $\lambda$ and $\gamma$ are real parameters. This is an exactly solved model. By using the Jordan Wigner transformation, the Hamiltonian is formally equivalent to the quasi-free fermion one. More precisely, consider the formal transformation

$$
a_{j}^{*}=\sigma_{+}^{(j)} \prod_{k=-\infty}^{j-1} \sigma_{z}^{(k)}, \quad a_{j}=\sigma_{-}^{(j)} \prod_{k=-\infty}^{j-1} \sigma_{z}^{(k)} .
$$

Then $a_{j}^{*}$ and $a_{j}$ satisfy the canonical anti-commutation relations

$$
\left\{a_{j}^{*}, a_{k}^{*}\right\}=\left\{a_{j}, a_{k}\right\}=0, \quad\left\{a_{j}^{*}, a_{k}\right\}=\delta_{j, k} 1 .
$$

The Hamiltonian (3.2) of the XY model is bilinear in $a_{j}^{*}$ and $a_{k}$. We can give a mathematical meaning of the Jordan Wigner transformation for infinite systems (see [4]). The model has the unique ground state with gapless spectrum if $|\lambda|=1$ or if $\gamma=0$ and $|\lambda|<1$. Araki has constructed non-type I infrared representations for the XY model. The von Neumann algebra generated by $\mathcal{A}$ can be any hyperfinite factor type $I, I I$ and $I I I_{\lambda}(0<\lambda \leq 1)$.

We can also construct the infrared representation for the ferromagnetic isotropic Heisenberg model $(\Delta=1)$ XXX model using only product states. Here we sketch the 
construction. The Hamiltonian is now determined by the following equation

$$
H=\sum_{j \in \mathbf{Z}}\left\{1-\left(\sigma_{x}^{(j)} \sigma_{x}^{(j+1)}+\sigma_{y}^{(j)} \sigma_{y}^{(j+1)}+\sigma_{z}^{(j)} \sigma_{z}^{(j+1)}\right)\right\}
$$

We define the positive operator $h_{j}^{\mathrm{XxX}}$ by

$$
h_{j}^{\mathrm{XXX}}=\left\{1-\left(\sigma_{x}^{(j)} \sigma_{x}^{(j+1)}+\sigma_{y}^{(j)} \sigma_{y}^{(j+1)}+\sigma_{z}^{(j)} \sigma_{z}^{(j+1)}\right)\right\} \geq 0 .
$$

Koma and Nachtergaele proved that any ground state $\varphi$ of the model satisfies the zero energy condition (2.4) (cf. [13])

$$
\varphi\left(h_{j}^{\mathrm{XXX}}\right)=0 \quad \forall j \in \mathbf{Z} .
$$

This zero energy condition implies that the pure ground state is a translation invariant product state. Thus a pure ground state is parameterized by a unit vector in the two dimensional Hilbert space. Let $\varphi_{\xi}$ be the pure ground state of the ferromagnetic isotropic Heisenberg model specified by the condition

$$
\varphi_{\xi}\left(Q^{(j)}\right)=(\xi, Q \xi) .
$$

We consider $\varphi_{ \pm}=\varphi_{\xi_{ \pm}}$determined uniquely by

$$
\varphi_{ \pm}\left(\sigma_{z}^{(j)}\right)= \pm 1
$$

for any $j$ in $\mathbf{Z}$. Equivalently, $\xi_{ \pm}$is the vector such that

$$
\sigma_{z}^{(j)} \xi_{ \pm}= \pm \xi_{ \pm}
$$

$\varphi_{ \pm}$is the state with all the spin in $\pm \mathrm{z}$ direction.

Next, we introduce the local gauge transform $V_{k}(k \in \mathbf{Z})$ which flips the spin at some point of lattice with a cost of small energy. First, we set

$$
p(k)=\sum_{j=1}^{k} j^{3}=\frac{k^{2}(k+1)^{2}}{4} .
$$

Then

We consider

$$
V_{k}=\exp \left(i \frac{2 \pi}{k^{3}} \sum_{j=p(k)}^{p(k+1)-1}(j-p(k)) \sigma_{z}^{(j)}\right)
$$

where

$$
\tilde{\varphi}_{ \pm}(Q)=\lim _{l \rightarrow \infty} \varphi_{ \pm} \circ \operatorname{ad}\left(\prod_{k=2}^{l} V_{k}\right)(Q), \quad Q \in \mathcal{A}
$$

$$
\operatorname{ad}\left(\prod_{k=2}^{l} V_{k}\right)(Q)=\left(\prod_{k=2}^{l} V_{k}\right) Q\left(\prod_{k=2}^{l} V_{k}\right)^{-1} .
$$

Each adjoint action of $V_{k}$ creates a state with a reversed spin. The cost of the energy is proportional to $\frac{1}{k^{2}}$ so that we get $\tilde{\varphi}_{ \pm}$with a finite loss of energy. As $\tilde{\varphi}_{ \pm}$contains infinitely many reversed spins the representation is not equivalent to any ground state.

Proposition 3.1. The GNS representation associated with $\tilde{\varphi}_{ \pm}$is a positive energy representation which is disjoint from any ground state representation of the isotropic ferromagnetic Heisenberg model. 
We can construct many mutually disjoint infrared representations for the above example in the same manner. We feel that the set of infrared representations is too large to be described in a compact form and this is always the case for Hamiltonians with gapless spectra.

4. Soliton sectors. Suppose we have two pure translation invariant ground states $\varphi_{ \pm}$. Consider the situation where any unit vector $\xi$ in another representation $\{\pi, \mathcal{H}\}$ satisfies the following asymptotic property

$$
\lim _{j \rightarrow \pm \infty}\left(\xi, \pi\left(\tau_{j}(Q)\right) \xi\right)=\varphi_{ \pm}(Q) .
$$

The state satisfying (4.1) may be regarded as the (classical) soliton-like object which interpolates two different vacuum by the tunneling effect. So by soliton sector, we mean a sector which connects two mutually non equivalent ground states. Obviously the sector satisfying (4.1) is constructed by the GNS representation associated with the state $\varphi_{-(-\infty,-1]} \otimes \varphi_{+[0, \infty)}$. Theorem 5.4 implies that positive energy representations which contain non-translation invariant ground state vector should be of this form if the Hamiltonian has the spectral gap.

Constructing non-translation invariant ground states for a translation invariant Hamiltonian is not an easy task. No criterion for the existence of non-translation invariant ground states is known at the moment. In principle, it is easier to obtain candidates of positive energy representations which contain non-translation invariant ground state vectors due to Theorem 5.4. The problem of searching non-translation invariant ground states is reduced to that of perturbation of point of spectrum. We explain the situation more explicitly in the case of ferromagnetic XXZ models with a large anisotropy $(\Delta>>1)$. The existence of the non-translation invariant ground state is verified from consideration of symmetry. The Hamiltonian we consider is presented in a slightly different but still equivalent form

$$
H=\sum_{j \in \mathbf{Z}}\left\{1-\sigma_{z}^{(j)} \sigma_{z}^{(j+1)}+\frac{1}{\Delta}\left(\sigma_{x}^{(j)} \sigma_{x}^{(j+1)}+\sigma_{y}^{(j)} \sigma_{y}^{(j+1)}\right)\right\}
$$

with $\Delta>1$. Any factor positive energy representation for the model is obtained by the GNS construction associated with either $\varphi_{ \pm}$of (3.5), $\psi_{K}$ or $\psi_{A K}$ where $\psi_{K}$ and $\psi_{A K}$ are defined by

$$
\psi_{K}=\varphi_{-(-\infty,-1]} \otimes \varphi_{+[0, \infty)}, \quad \psi_{A K}=\varphi_{+(-\infty,-1]} \otimes \varphi_{-[0, \infty)} .
$$

We consider the sector associated with $\psi_{K}$. Let $\left\{\pi_{K}, \mathcal{H}_{K}, \Omega_{K}\right\}$ be the GNS triple for $\psi_{K}$ where $\Omega_{K}$ is the GNS cyclic vector. It is known that this representation contains the non-translation invariant ground state vectors ([9]). These non-translation invariant ground states were discovered in connection with the quantum group $S U_{q}(2)$ symmetry. An example of non-translation invariant ground states is

$$
\Omega_{K}=\bigotimes_{j=-\infty}^{\infty} \frac{1}{\sqrt{1+q^{2 j}}}\left(\begin{array}{c}
1 \\
q^{j}
\end{array}\right)
$$

where $\Delta=1 / 2\left(q+q^{-1}\right), 0<q<1$. However we can prove the existence of non-translation 
invariant ground state vector without handling $S U_{q}(2)$ symmetry. Consider the following perturbed XXZ Hamiltonian $\tilde{H}_{n}(n>0)$ defined by

$$
\tilde{H}_{n}=\left(1-\frac{1}{\Delta}\right) \tilde{H}_{n}^{\text {Ising }}+\frac{1}{\Delta} \sum_{|j| \leq n, n \neq-1} h_{j}^{\mathrm{xxx}} \geq 0
$$

where $h_{j}^{\mathrm{XXX}}$ is defined in (3.4) and

$$
\tilde{H}_{n}^{\text {Ising }}=\left(1-\frac{1}{\Delta}\right) \sum_{j=-n}^{n}\left(1-\sigma_{z}^{(j)} \sigma_{z}^{(j+1)}\right)+\left(\sigma_{z}^{(-n)}-\sigma_{z}^{(n+1)}\right) \geq 0 .
$$

$\psi_{K}$ is a pure zero energy state for both $\tilde{H}_{n}$ and $\tilde{H}_{n}^{\text {Ising }}$, i.e. for any $n>0$

$$
\psi_{K}\left(\tilde{H}_{n}\right)=\psi_{K}\left(\tilde{H}_{n}^{\text {Ising }}\right)=0 .
$$

The following limit exists in the sense of the strong resolvent convergence

$$
\lim _{n \rightarrow \infty} \pi_{K}\left(\tilde{H}_{n}\right)=\tilde{H} \geq 0, \quad \lim _{n \rightarrow \infty} \pi_{K}\left(\tilde{H}_{n}^{\text {Ising }}\right)=\tilde{H}^{\text {Ising }} \geq 0 .
$$

Both $\tilde{H}$ and $\tilde{H}^{\text {Ising }}$ are positive operators with the spectral gap. The gap of $\tilde{H}$ is bounded below by $2\left(1-\frac{1}{\Delta}\right)$. The Hamiltonian $H$ of the (translation invariant) original model can be defined as the bounded perturbation of $\tilde{H}$.

Set

$$
S_{z}=\sum_{j<0}\left(1+\sigma_{z}^{(j)}\right)-\sum_{j \geq 0}\left(1-\sigma_{z}^{(j)}\right) .
$$

$S_{z}$ is well defined on $\left\{\pi_{K}(), \mathcal{H}_{K}\right\}$ and commutes (strongly) with $\tilde{H}, H$ and $\tilde{H}^{\text {Ising }}$. Our aim is to establish the existence of point spectrum at the bottom of spectra of $H$. It suffices to show the same claim in all the subspaces $\mathcal{H}_{s}$ of the $S_{z}$ spin $=$ s.

$$
\mathcal{H}_{0}=\left\{\eta \in \mathcal{H}_{K} \mid S_{z} \eta=s\right\} .
$$

Thus we consider the reduction of the Hamiltonian $\tilde{H}$ on the subspace $\mathcal{H}_{0}$. (The reduction to other spaces $\mathcal{H}_{s}$ can be handled in the same way.) It is straightforward to show that the GNS cyclic vector $\Omega_{K}$ is the unique vector (up to scalar) satisfying

$$
\tilde{H} \Omega_{K}=0, \quad S_{z} \Omega_{K}=0 .
$$

Due to presence of spectral gap for $\tilde{H}$ we can apply the analytic perturbation theory for the ground state of the perturbed Hamiltonian $\tilde{H}(\delta)$ defined by

$$
\tilde{H}(\delta)=\tilde{H}+\delta h_{-1}^{\mathrm{xxx}} .
$$

It turns out that the ground state (the point spectrum in the bottom of spectra) of $\tilde{H}(\delta)$ exists in $\mathcal{H}_{0}$. Moreover, if $\Delta$ is sufficiently large we can set $\Delta=\delta$ which implies the existence of the non-translation invariant ground state. We can continue the same procedure as far as the spectral gap is open and we obtain the non-translation invariant ground state for any $\Delta>1$.

The above argument shows that static soliton sectors (soliton sector with time invariant vectors) exist for the ferromagnetic XXZ model with spin $1 / 2$. We have not carried out any computation to arrive at the conclusion. We expect that the argument itself may be valid for more complicated systems. 
5. Complete list of positive energy representations. The aim of this section is to present a complete list of positive energy representations. We assume that Hamiltonians have the spectral gap. We also assume another condition. To state it we fix notations.

Consider $H_{\Lambda}$ of (1.2). Let $E_{\Lambda}$ be the smallest eigenvalue for $H_{\Lambda}$. Set

$$
e_{\infty}=\inf \psi\left(h_{0}\right)
$$

where the infimum is taken among all translation invariant states. It is known that $e_{\infty}$ is attained by a translation invariant state $\varphi, e_{\infty}=\varphi\left(h_{0}\right)$, if and only if $\varphi$ is a ground state (see [5]).

In what follows, a subset $\Lambda$ of $\mathbf{Z}$ will be an interval $\Lambda=[n, m]=\{n, n+1, n+2, \ldots, m\}$. The $\operatorname{limit}_{\Lambda \rightarrow \mathbf{Z}}$ means $n \rightarrow-\infty, m \rightarrow \infty$.

Assumption 5.1. The following limit exists,

$$
\lim _{\Lambda \rightarrow \mathbf{Z}}\left|E_{\Lambda}-\right| \Lambda\left|e_{\infty}\right|=0
$$

for $\Lambda=[n, m]$.

Remark 5.2. Assumption 5.1 is satisfied for the Hamiltonian with the zero energy state. In fact, the existence of the zero energy state implies that $E_{\Lambda}=|\Lambda| e_{\infty}$. We can also verify Assumption 5.1 for certain models by the cluster expansion technique, e.g. for the XYZ model

$$
H=\sum_{j \in \mathbf{Z}}\left\{\sigma_{x}^{(j)} \sigma_{x}^{(j+1)}+\varepsilon_{1} \sigma_{y}^{(j)} \sigma_{y}^{(j+1)}+\varepsilon_{2} \sigma_{z}^{(j)} \sigma_{z}^{(j+1)}\right\}
$$

with large anisotropy, i.e. such that $\left|\varepsilon_{1}\right|<<1$ and $\left|\varepsilon_{2}\right|<<1$.

On the other hand, for solvable massless models, the above assumption may not be valid. It seems that

$$
E_{\Lambda} \sim|\Lambda| e_{\infty}+|\partial \Lambda| c
$$

where $c \neq 0$ may be determined by the conformal field theory.

The next assumption is the existence of spectral gap uniformly in $\Lambda$.

Assumption 5.3. There exists a positive number $\gamma>0$ independent of $\Lambda$ and there are $\delta_{\Lambda}>0$ such that

and

$$
\lim _{\Lambda \rightarrow \mathbf{Z}} \delta_{\Lambda}=0
$$

$$
\operatorname{spec}\left(H_{\Lambda}\right) \cap\left(E_{\Lambda}+\delta_{\Lambda}, E_{\Lambda}+\delta_{\Lambda}+\gamma\right)=\emptyset .
$$

Note that we assume the uniform spectral gap for $H_{\Lambda}$ and we do not assume anything for the Hamiltonian with an additional boundary term.

The following theorem is the extension of the main result of our previous paper [14].

TheOREm 5.4. Suppose that Assumption 5.1 and 5.3 are valid. Let $\pi$ be a factor positive energy representation of $\mathcal{A}$ on $\mathcal{H}$. Then $\pi$ is quasi-equivalent to a pure ground state representation $\pi_{g}$ for the Hamiltonian $H-H_{\Lambda_{0}}$ with some $\Lambda_{0}=[-n, n]$.

$$
H-H_{\Lambda_{0}}=\sum_{|j|>n-r} h_{j}
$$


where $r$ is the range of interaction. Moreover the pure ground state $\varphi_{g}$ in $\pi_{g}$ is obtained by the thermodynamic limit of a sequence of finite volume ground states $\varphi_{\Lambda_{n}}$ of $H_{\Lambda_{n}}$.

$$
\begin{gathered}
\varphi_{\Lambda_{n}}\left(Q^{*}\left[H_{\Lambda_{n}}, Q\right]\right) \geq 0, \quad Q \in \mathcal{A}_{\Lambda_{n}}, \\
\varphi_{g}(Q)=\lim _{n \rightarrow \infty} \varphi_{\Lambda_{n}}(Q), \quad Q \in \mathcal{A} .
\end{gathered}
$$

The result is rephrased as follows. Consider the half infinite Hamiltonians $H_{[0, \infty)}$ and $H_{(-\infty,-1]}$. Let $G^{(+)}$be the set of all ground state representations for $H_{[0, \infty)}$ obtained from the thermodynamic limit. Let $G^{(+)}$be the set of all ground state representations for $H_{(-\infty,-1]}$. Any positive energy representation is quasi-equivalent to a subrepresentation in the universal positive energy representation $\tilde{\pi}$ on $\mathcal{H}$ defined by

$$
\{\tilde{\pi}, \mathcal{H}\}=\sum\left\{\oplus \pi_{-} \otimes \pi_{+}, \oplus \mathcal{H}_{-} \otimes \mathcal{H}_{+}\right\}
$$

where the direct sum is taken for all representations $\left\{\pi_{+}, \mathcal{H}_{+}\right\}$in $G^{(+)}$and $\left\{\pi_{-}, \mathcal{H}_{-}\right\}$in $G^{(-)}$. If an infinite number of equivalence classes of ground state representations in $G^{(+)}$ or in $G^{(-)}$exists, the above statement should be corrected suitably (direct integral etc.).

As a corollary to our theorem for positive energy representations, we obtain that the strong resolvent convergence of $H_{\Lambda}-E_{\Lambda} 1$ to $H_{\pi}$. Thus we obtain the following result.

Corollary 5.5. Suppose that Assumption 5.1 and 5.3 are valid. Let $\pi$ be a (not necessarily translation invariant) ground state representation on $\mathcal{H}$ and let $H_{\pi}$ be the positive selfadjoint operator satisfying (1.1). Then for $\gamma>0$ of Assumption 5.3,

$$
\left(\inf H_{\pi}, \inf H_{\pi}+\gamma\right) \cap \operatorname{spec}\left(H_{\pi}\right)=\emptyset .
$$

The exact gap of the spectrum may depend on choice of the representation $\pi$. Suppose $\pi$ is a non-translation invariant ground state such that $\pi=\pi_{-} \otimes \pi_{+}$for some half infinite ground state representations $\pi_{-}$and $\pi_{+}$. The spectral gap is bounded below by that of $H_{\pi_{-}}$and $H_{\pi_{+}}$.

In the case of the XYZ model (5.3) with sufficiently small $\left|\varepsilon_{1}\right|$ and $\left|\varepsilon_{2}\right|$, we can check our assumptions with the aid of expansion techniques. Even though the model is solvable we are not certain that the solution is complete and we may also have other difficulties in taking the thermodynamic limit so we employed expansion techniques. There are four equivalence classes of positive energy representations. In fact, by using expansion techniques, we can show that there exist precisely two periodic pure ground states $\varphi_{\text {even }}$ and $\varphi_{\text {odd }}$ such that $\varphi_{\text {even }}=\varphi_{\text {odd }} \circ \tau_{1}$ and any periodic ground state is a convex combination of $\varphi_{\text {even }}$ and $\varphi_{\text {odd }}$. Other two sectors are obtained by the GNS representation for $\psi_{K}$ or $\psi_{A K}$ where

$$
\psi_{K}=\left.\left.\varphi_{\text {even }}\right|_{(-\infty,-1]} \otimes \varphi_{\text {odd }}\right|_{[0, \infty)}, \quad \psi_{A K}=\left.\left.\varphi_{\text {odd }}\right|_{(-\infty,-1]} \otimes \varphi_{\text {even }}\right|_{[0, \infty)} .
$$

An open question is whether non periodic ground states for the model exist. The problem is equivalent to showing that the bottom of the spectrum of $H_{\pi}$ is in these sectors.

Acknowledgments. The work is supported by Sumitomo Foundation, Japan. The result of this paper was announced at the conference on quantum probability held in Gdańsk, July 1997. The author would like to acknowledge the warm hospitality of the organizers of the conference. 


\section{References}

[1] I. Affleck, T. Kennedy, E. H. Lieb and H. Tasaki, Valence Bond Ground States in Isotropic Quantum Antiferromagnets, Commun. Math. Phys. 115 (1988), 477-528.

[2] S. R. Alcaraz, R. S. Salinas and W. F. Wreszinski, Anisotropic ferromagnetic quantum domain, Phys. Rev. Lett. 75 (1995), 930-933.

[3] H. Araki, Soliton sector of the XY-model, International Journal of Modern Physics B 10, Nos 13 \& 14 (1996), 1685-1693.

[4] H. Araki and T. Matsui, Ground states of the XY model, Commun. Math. Phys. 101 (1985), 213-245.

[5] O. Bratteli, A. Kishimoto and D. Robinson, Ground states of quantum spin systems, Commun. Math. Phys. 64 (1978), 41-48.

[6] O. Bratteli and D. Robinson, Operator algebras and quantum statistical mechanics II, Springer, 1997.

[7] M. Fannes, B. Nachtergaele and R. Werner, Finitely Correlated States on Quantum Spin Chains, Commun. Math. Phys. 144 (1992), 443-490.

[8] J. Frölich, New superselection sectors (soliton states) in two dimensional Bose quantum field theory, Commun. Math. Phys. 47 (1976), 269-310.

[9] C. T. Gottstein and R. Werner, Zero-energy states of the ferromagnetic XXZ chain, preprint, Osnabrück 1995.

[10] C. T. Gottstein and R. Werner, Zero-energy ground states of quantum lattice systems, preprint, Osnabrück 1995.

[11] R. Haag, Local Quantum Physics, Springer-Verlag, 1992.

[12] T. Koma and B. Nachtergaele, The spectral gap of the ferromagnetic XXZ chain, Lett. Math. Phys. 40 (1997), 1-16.

[13] T. Koma and B. Nachtergaele, preprint.

[14] T. Matsui, Translationally Symmetry Breaking and Soliton Sectors for Massive Quantum Spin Models in $1+1$ Dimensions, Commun. Math. Phys. 189 (1997), 127-144.

[15] T. Matsui, On the spectrum of the kink of the ferromagnetic XXZ model, to appear in Lett. Math. Phys.

[16] T. Matsui, On ground states of the one-dimensional ferromagnetic $X X Z$ model, Lett. Math. Phys. 37 (1996), 397-403.

[17] D. Schlingemann, On algebraic theory of soliton and anti-soliton sectors, Rev. Math. Phys. 8 (1996), 301-326. 